\title{
Investigation of Bush Rotation Speed of Floating Bush Bearings by Oil Film Observation Using X-ray CT
}

\author{
Shinpei Kotani ${ }^{1)^{*}}$ and Masayuki Ochiai ${ }^{2)}$ \\ ${ }^{1)}$ Graduate School of Engineering, Tokai University, 4-1-1 Kitakaname, Hiratsuka, Kanagawa 259-1292, Japan \\ ${ }^{2)}$ Department of Mechanical Engineering, Tokai University, 4-1-1 Kitakaname, Hiratsuka, Kanagawa 259-1292, Japan \\ *Corresponding author: Shinpei Kotani (shinpei.k.tokaiuni@gmail.com)
}

Manuscript received 03 July 2019; accepted 16 September 2019; published 15 December 2019

Presented at the International Tribology Conference Sendai 2019, 17-21 September, 2019

\begin{abstract}
A floating bush bearing has a double oil film, which is difficult to observe using traditional methods. In this study, the oil film of a floating bush bearing is observed using X-ray computer tomography. By appropriately processing the acquired CT images, double oil films can be observed efficiently. The CT images show that the oil film area decreases in the inner clearance and does not change in the outer clearance when the increase of the bush rotation speed is suppressed despite the increase in the shaft rotation speed. This phenomenon can be explained by the oil film area of the inner clearance decreasing under the influence of the centrifugal force acting on the oil film. When there is enough oil in the inner clearance, it is observed that the bush rotation speed increases linearly. These results indicate that the bush rotation speed is closely related to the oil film area of the inner clearance.
\end{abstract}

\section{Keywords}

floating bush bearing, oil film observation, X-ray computer tomography, bush rotation speed, fluid lubrication, CT images, sliding bearing, centrifugal force, visualization

\section{Introduction}

A floating bush bearing is a type of sliding bearing that is used in turbochargers and operated at extremely high speeds. As shown in Fig. 1, this type of bearing has a cylinder with oil holes, called a floating bush, between the shaft and the bearing. In operation, the shear force of the oil film rotates the bush and forms a dual oil film, which supports the load. One oil film forms in the clearance between the shaft and the bush (called inner clearance) and the other forms in the clearance between the bush and the bearing (called outer clearance).

The conventional lubrication theory governing the stability of floating bush bearings is based on the Reynolds equation, which predicts that a rotor supported by floating bush bearings is stable at low speed and unstable at high speed [1-3]. In addition, the bush rotation speed is determined by the balance of the viscous friction forces that act on the inside and outside of the floating bush, and tends to increase linearly with increasing shaft rotation speed [4-7]. However, it has been reported that rotors supported by floating bush bearings are stable at high speeds; furthermore, for rotation speeds at high, the increase in the bush rotation speed is suppressed and the speed remains constant [8-11]. Several studies have been conducted to explain

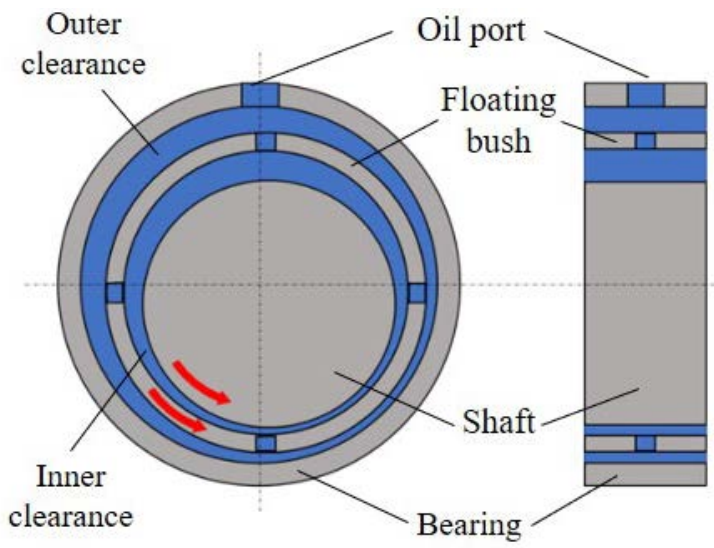

Fig. 1 Outline drawing of floating bush bearing

this suppression phenomenon, but the cause has not yet been clarified.

Hatakenaka et al. [12] stated that the suppression of the bush rotation speed is due to the centrifugal force acting on the oil film and creating a pressure difference in the inner clearance, which results in cavitation and reduction of the oil film area. 
This has been confirmed by observation of the oil film in a plain bearing that supports a high-speed rotating shaft [13], but not in a floating bush bearing. In contrast, Tsuruta [11] stated that reduction in the oil film area of the inner clearance is caused by the lack of refueling of the inner clearance due to centrifugal force. However, evidence to support this explanation has also not been obtained. Furthermore, while Wang et al. [14] calculated the oil film area when the increase in the bush rotation speed was suppressed by fluid simulation, the validity of the results was not verified by comparison with the oil film area of the actual floating bush bearing. On the other hand, in a study that observed the oil film of a floating bush bearing, an oil film on the inner clearance was examined by Tsuda [15] using an endoscopic camera. However, this experiment used the bush rotation speed as a parameter; the shaft was not rotating. Therefore, the applicable experimental conditions were not reproduced. Overall, the bush rotation speed suppression phenomenon has been studied using various approaches, but the oil film has not been adequately observed, and the experimental evidence of any single explanation is insufficient.

This study was aimed at investigating the suppression of the increase in the bush rotation speed in order to elucidate the cause of the phenomenon. Visualization of the oil film is complicated owing to the properties of the bearing because of the following reasons. First, in order to observe the oil film, it is necessary to simultaneously observe the clearance between the inner and outer sides of the bush. This is because the rotational torque of the floating bush bearings is determined by the balance between the driving torque, which arises due to the shearing of the oil film in the inner clearance, and the braking torque, which arises due to the shearing of the oil film in the outer clearance. Second, the operating floating bush bearing has a double oil film, because of which the oil film images interfere with each other and make it difficult to observe with conventional visualization methods [13, 16-18].

$\mathrm{X}$-ray computer tomography (X-ray CT) was used for this observation. As this method is usable regardless of the structure of the object, it can be used as an innovative observation method for the double oil film of a floating bush bearing. To observe the oil film, a bearing test equipment that enables oil film observation by X-ray CT was made specifically for use in this study. Then, the bush rotation speed was measured and compared with the oil film area, and an attempt was made to comprehend the experimental results.

\section{Experimental setup}

\subsection{X-ray CT}

A Nikon industrial X-ray CT XT H 225 (Control software inspect- $X$ ) was used to examine the oil film in this study. This device acquires images of the entire circumference of an object via X-ray irradiation in all directions at regular intervals while the object undergoes a $360^{\circ}$ rotation. When $X$-rays pass through the object, attenuation of the $\mathrm{X}$-ray intensity $I_{1}$ is expressed by the following Eq. (1).

$$
I_{1}=I_{0} \exp (-\eta X)
$$

where $I_{0}, \eta$, and $X$ represent the incident $X$-ray intensity, linear absorption coefficient of the transmission material, and transmission distance, respectively. These values are affected by the characteristics of the experimental conditions. For instance, the linear absorption coefficient $\eta$ depends on the density and atomic number of the object, as well as the X-ray intensity.
The incident X-ray intensity is strongly influenced by the material and structure of the object. Additionally, the value of attenuation is proportional to the transmission distance, which is extremely short because there is even a thin bearing clearance. The detection and quantification of the attenuation can be optimized by reducing the incident $X$-ray intensity, selecting a bearing material that easily transmits X-rays, limiting X-ray transmission, and adjusting the $\mathrm{X}$-ray irradiation range.

\subsection{CT image reconstruction procedure}

In this study, 500 projections obtained via X-ray imaging were converted into volume data using the reconfiguration software CT pro 3D and observed as CT images by the 3D object analysis software My VGL 3.1. Volume rendering is a reconstruction method that provides both the surface information and internal information of objects. In this method, the opacity depends on the continuous change in the $\mathrm{CT}$ value, which represents the degree of X-ray absorption of the substance. As a result, the object is illustrated by shading indicative of light transmission and reflection. By adjusting the opacity, which is a measure of the impenetrability of light, the CT images of an object can be made translucent. Therefore, the CT images reconstructed by this method are suitable for observing the inside of an object composed of multiple substances.

\subsection{Experimental equipment}

As shown in Fig. 2, the motor in the experimental rig is controlled by a motor driver, and the power of the motor is transmitted to the upper acrylic shaft, which is supported by the floating bush bearing through a pulley and a belt. Therefore, a load of $12 \mathrm{~N}$ is applied to the bearing due to belt tension. By adopting such a structure as shown in Fig. 2, X-rays can be transmitted only through the floating bush bearing by adjusting the irradiation range. Under these conditions, X-rays do not pass through metal parts such as motors; hence, an increase in incident X-ray intensity can be avoided. Furthermore, as the incident $X$-ray intensity depends on the material through which the $\mathrm{X}$-rays penetrate, the floating bush bearing and the bearing stand are made of acrylic and ABS resin. Figure 3 and Table 1 illustrate the experimental floating bush bearing. The floating bush bearing was provided with four oil holes at $90^{\circ}$ intervals. As a lubricating method for the bearing, the lubricating oil leaking from the bearing side end was directed into an oil tank, and the oil from the tank was pumped up to the bearing. With this pump, the refueling condition during X-ray imaging

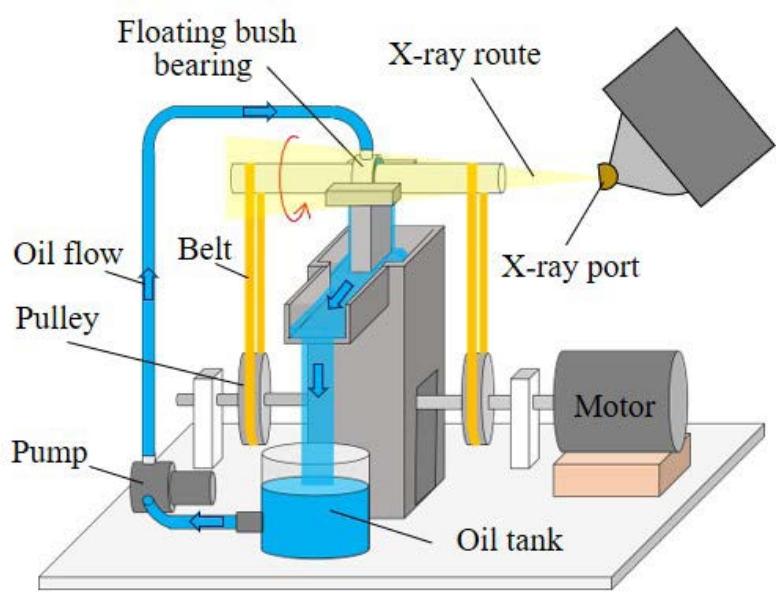

Fig. 2 Illustration of experimental test rig 


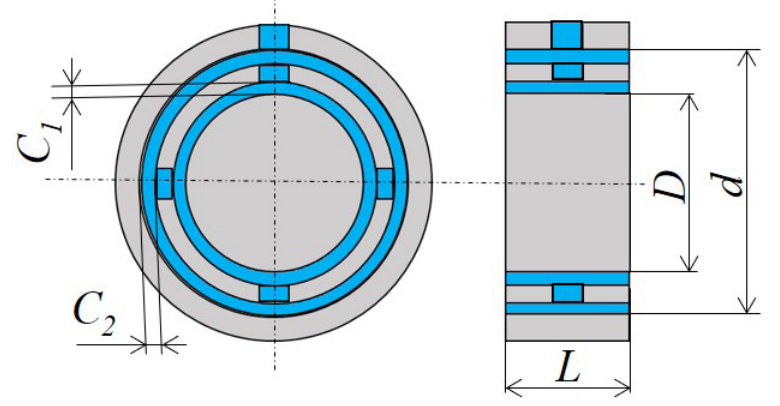

Fig. 3 Illustration of floating bush bearing

Table 1 Dimensions of test bearing

\begin{tabular}{l|c}
\hline Shaft diameter $D[\mathrm{~mm}]$ & 20 \\
\hline Bearing inner diameter $d[\mathrm{~mm}]$ & 25 \\
\hline Inner clearance $C_{1}[\mu \mathrm{m}]$ & 100 \\
\hline Outer clearance $C_{2}[\mu \mathrm{m}]$ & 180 \\
\hline Bearing width $L[\mathrm{~mm}]$ & 14.5 \\
\hline \hline
\end{tabular}

is maintained at a constant oil supply pressure of $11 \mathrm{kPa}$ and supply rate of $3.4 \mathrm{~mL} / \mathrm{s}$. The oil used was a viscosity grade 32 industrial lubricant.

\subsection{Imaging of floating bush bearings in rotation by X-ray CT}

Figure 4 shows the test equipment installed for the $\mathrm{X}$-ray $\mathrm{CT}$. The power supply (a 24-volt battery) and the motor driver were fixed on an acrylic plate together with the aforementioned bearing test apparatus and installed on the X-ray CT machine. Typically, if an object moves during X-ray imaging, each acquired projection will change, making it impossible to construct a CT image. Thus, it is premised that the subject is stationary. However, as the oil film of a floating bush bearing is formed by the flow of oil that is only generated when the shaft rotates, it is necessary to image the flowing oil film. Therefore, the authors focused on the fact that the boundary between the oil film and the gas phase in the bearing clearance of journal bearings hardly changed in steady state. In this study, the boundary between the oil film and the gas phase is considered to be almost stationary in X-ray imaging during shaft rotation. Therefore, this boundary is reflected in the CT image reconstructed from the projections, enabling observation of the oil film and gas phase.

\subsection{Measurement of floating bush speed}

The floating bush was marked and photographed with a high-speed camera, and the rotation speed of the bush was calculated from the number of frame rates of the camera. This measurement was performed at shaft rotation speeds within the range of 7,000 rpm to $13,000 \mathrm{rpm}$. The refueling conditions were the same as those for $\mathrm{X}$-ray imaging.

\section{Results and discussion}

3.1 Establishment of oil film observation method using CT image

Figure 5 is a cross-sectional CT image when the shaft is rotated at 5,000 rpm without rotating the floating bush. The voxel size of this CT image was $31 \mu \mathrm{m}$. From this image, it was confirmed that enough resolution and contrast could be achieved by the experimental setup. During $X$-ray imaging, size of the focal spot on the X-ray port was $26 \mu \mathrm{m}$, and a $2.5 \mathrm{~mm}$ thick copper filter was used. This cut surface is shown in Fig. $5(a)$, and the cross section of this figure was located at the center of the bearing width $(Z=7.25 \mathrm{~mm})$. Figure $5(\mathrm{c})$ presents an enlarged view of region A where the boundary between the oil and gas phase shown in Fig. 5(b) can be viewed in detail. Figure

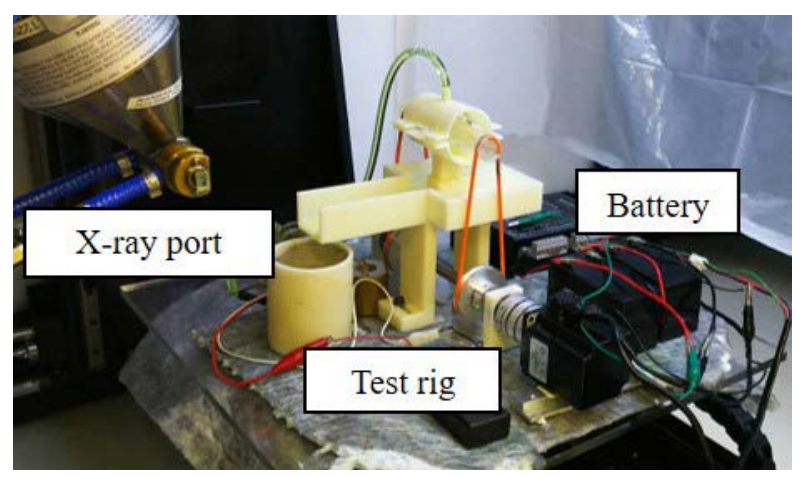

Fig. 4 Test equipment installed on X-ray CT

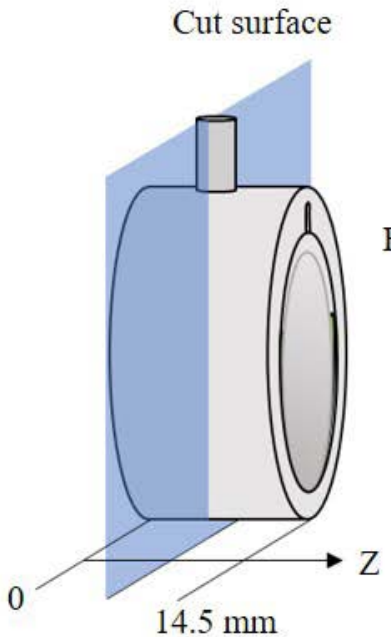

(a) Outline of cut surface

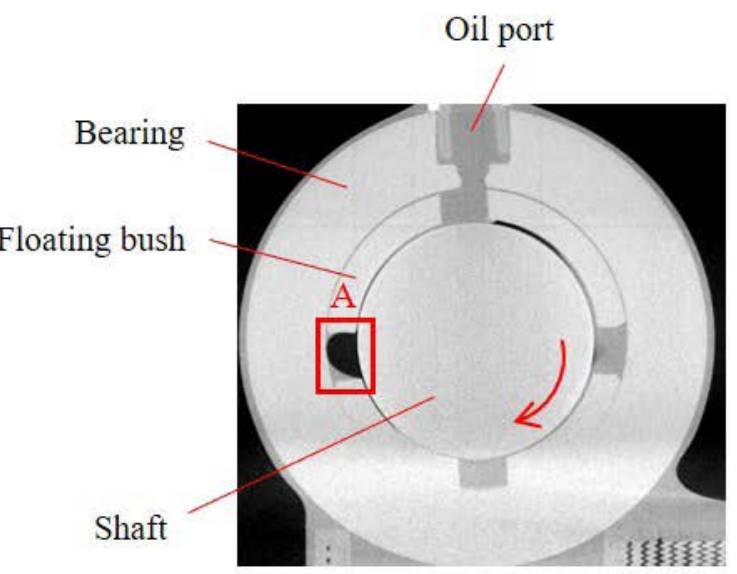

(b) CT image $(\mathrm{Z}=7.25 \mathrm{~mm})$

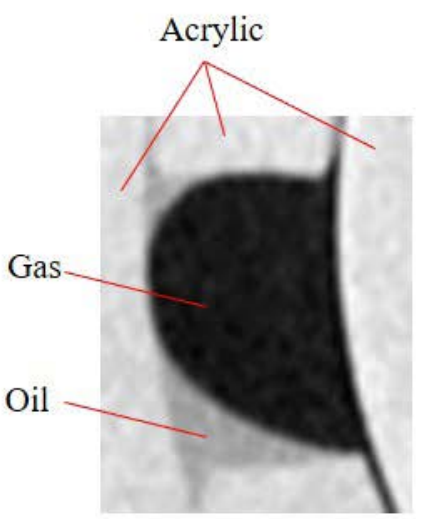

(c) Enlarged view of area A

Fig. 5 Cross-sectional CT image with shaft rotating at 5,000 rpm 
5(c) indicates that the oil film and the gas phase in the bearing clearance are observable using monochromic shades. These images confirm that the oil film in the minute bearing clearance can be observed in the CT image according to the design guideline of the test equipment used in this study. Figure 6 shows the three-dimensional image of Fig. 5. The CT image in Fig. 6(b) is adjusted so that the higher the CT value, the lower the opacity, and the lower the $\mathrm{CT}$ value, the higher the opacity. Therefore, acrylic and oils, having relatively higher $\mathrm{CT}$ values on the $\mathrm{CT}$ image, became translucent, and the gas phase, having lower CT values, was recolored to be white. As the bearing clearance is a gas-liquid two-phase flow, the oil film region can be rendered with this color correction method. In contrast, Fig. 7 shows the three-dimensional $\mathrm{CT}$ image of the case when the shaft is rotating at 10,000 rpm and the bush is also rotating. This image has already been adjusted for opacity. This figure shows that it is difficult to observe the oil film in the outer and inner clearance because the two oil films appear to overlap when the floating bush and shaft are both rotating. Therefore, the two clearances were separated in image processing by hollowing the inner clearance around the center of the bearing. Figure 8 shows the 3D CT image after hollowing out the inner clearance of the $\mathrm{CT}$ image in Fig. 7. This image processing allows simultaneous observation of both the outer and inner clearances. Hence, all subsequent oil film observations were performed by adjusting the opacity in this manner.

3.2 Oil film observation in the area where the increase in the bush rotation speed is suppressed

Figure 9 shows the relationship between the shaft rotation speed and bush rotation speed. This graph shows that the bush rotation speed is approximately constant between 10,000 and $13,000 \mathrm{rpm}$. Therefore, the oil film was observed at shaft rotation

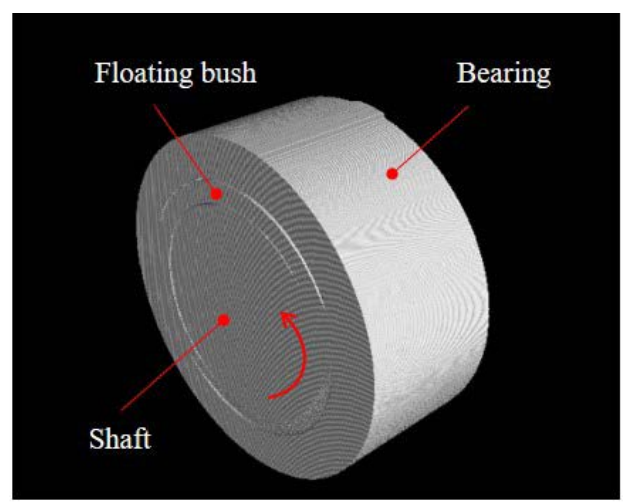

(a) Before opacity adjustment

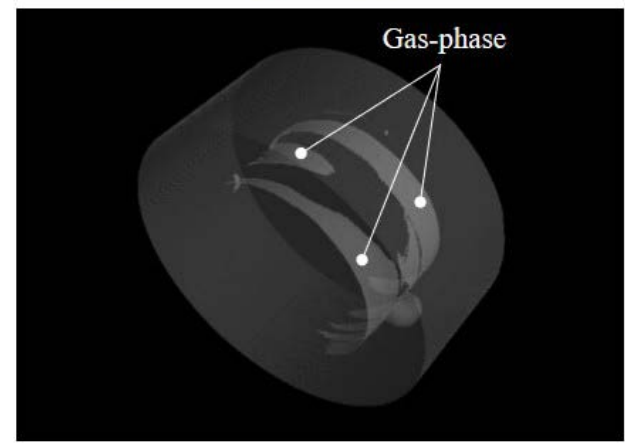

(b) After opacity adjustment

Fig. 6 3D CT image with shaft roating at 5,000rpm speeds of 10,000, 11,000, and 12,000 rpm in order to examine the suppression of the increase in the bush rotation speed. Figure 10 shows a CT image of a floating bush bearing at each shaft rotation speed. The voxel size of this CT image was $30 \mu \mathrm{m}$. In addition, during X-ray imaging, the size of the focal spot on the $X$-ray port was $27 \mu \mathrm{m}$, and a $2.5 \mathrm{~mm}$-thick copper filter was

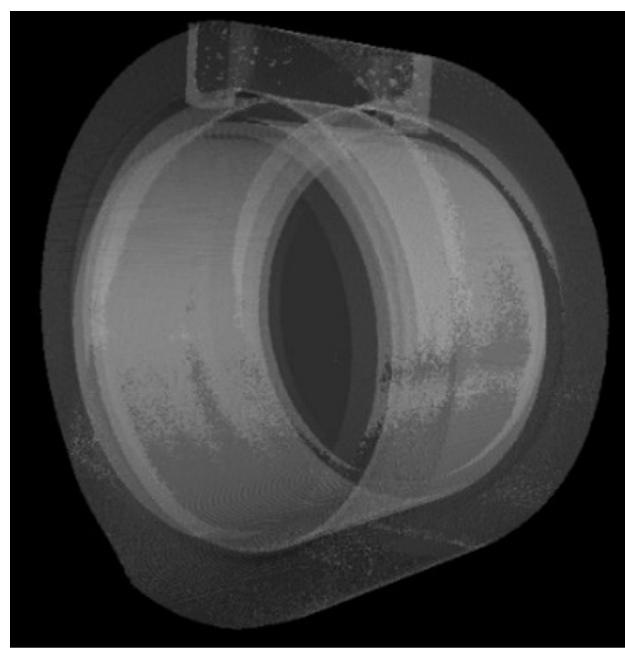

Fig. 7 3D CT image with rotating shaft and bush (Shaft rotation speed 10,000rpm)

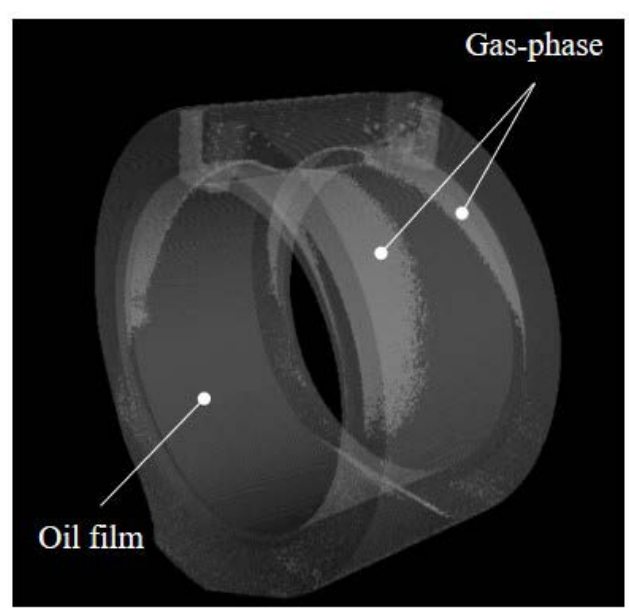

(a) Outer clearance

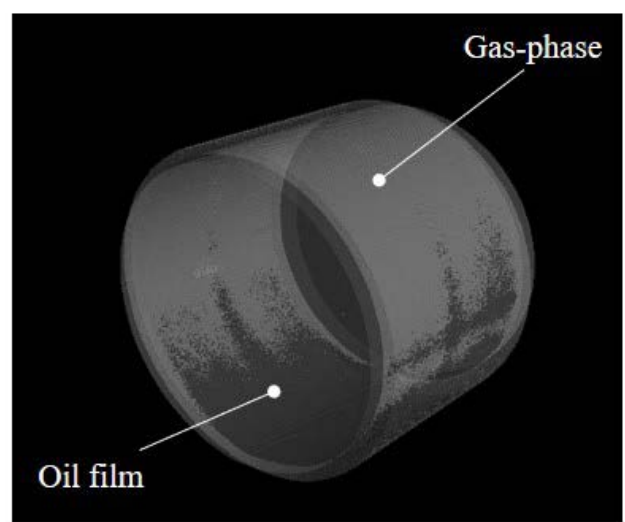

(b) Inner clearance

Fig. 8 3D CT image after separation (Shaft rotation speed 10,000rpm) 


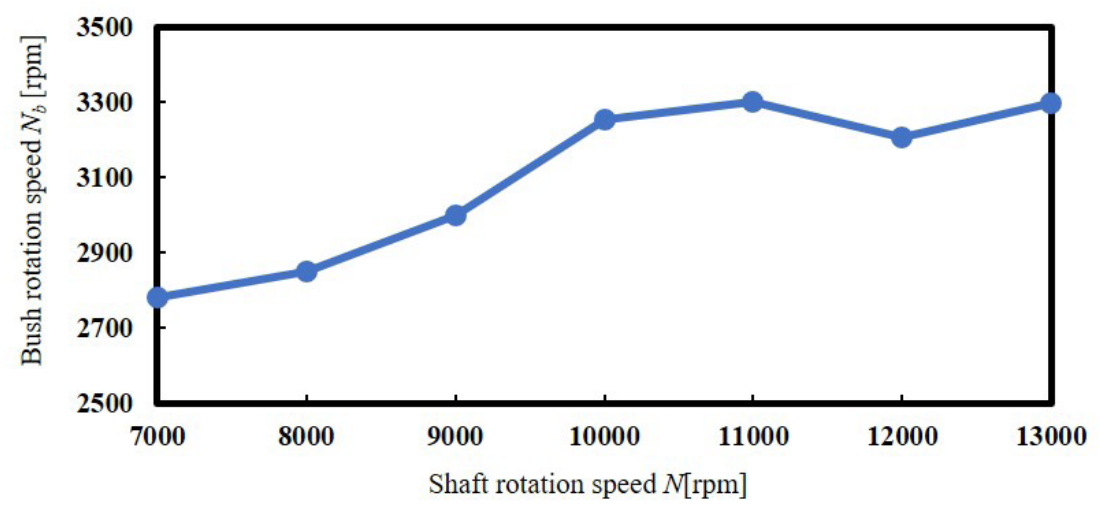

Fig. 9 Relationship between shaft rotation speed and bush rotation speed

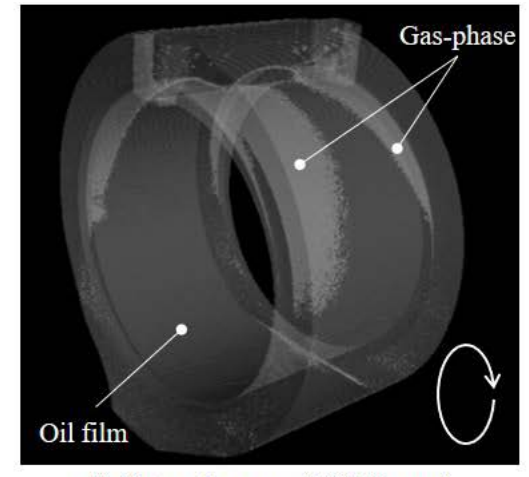

(i) Outer clearance $(10,000 \mathrm{rpm})$

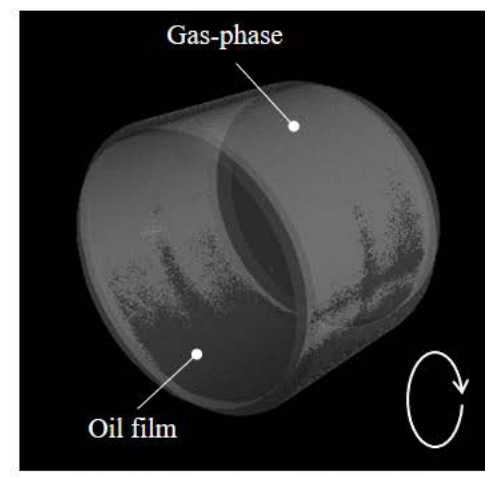

(ii) Inner clearance $(10,000 \mathrm{rpm})$

(a) Shaft rotation speed: $10,000 \mathrm{rpm}$

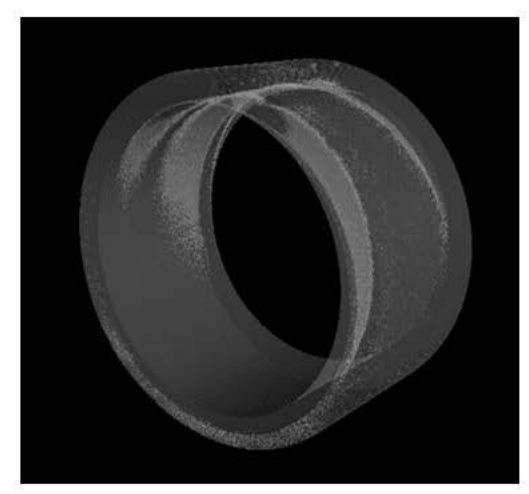

(iii) Outer clearance (11,000 rpm)

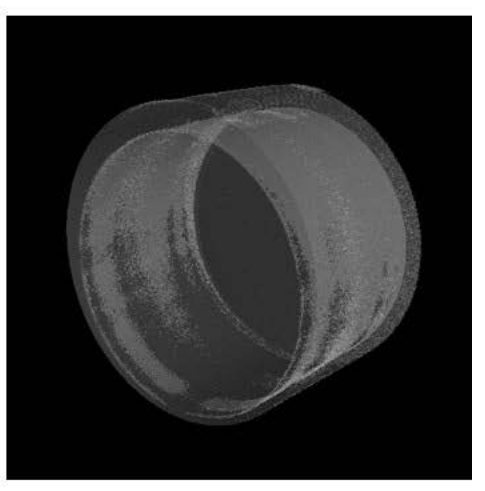

(iv) Inner clearance (11,000rpm)

(b) Shaft rotation speed: $11,000 \mathrm{rpm}$

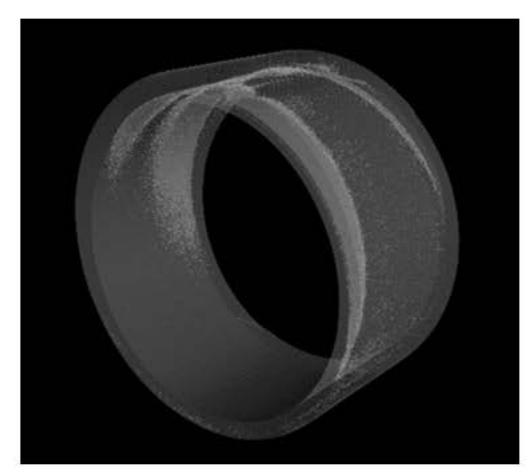

(v) Outer clearance $(12,000 \mathrm{rpm})$

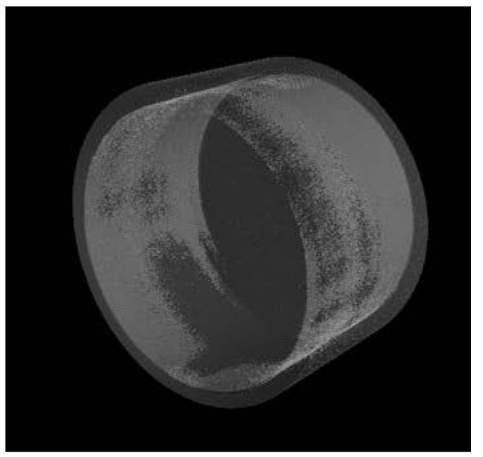

(vi) Inner clearance (12,000 rpm)

(c) Shaft rotation speed: $12,000 \mathrm{rpm}$

Fig. 10 CT image of suppression area 
attached to the X-ray port. This figure shows the differences in the oil film area between the inner and outer clearances. In other words, the oil films of the inner clearances observed at (i), (iii), and (v) in Fig. 10 were formed only on the lower halves of the bearings, but the oil films of the outer clearances observed at (ii), (iv), and (vi) in Fig. 10 were formed on the entire peripheries of the outer clearances. The oil film area of the inner clearance indicates that oil did not flow into the inner clearance from the oil port installed at the top of the bearing. This phenomenon is seemingly caused by the centrifugal force acting on the oil film by the rotation of the bush, preventing the oil from flowing from the oil port into the inner clearance. Tsuruta [11] had predicted this phenomenon from experimental results; however, this is the first time that it was confirmed through oil film observation. In addition, the oil inflow path of the inner clearance is considered as follows: the oil that did not flow directly into the inner clearance from the oil port due to centrifugal force flows into the outer clearance due to bush rotation. The pressure of the oil flowing into the outer clearance increases due to wedge action, and oil is supplied from the outer clearance to the inner clearance by this pressure.

Then, the clearance was compared using the CT image of each shaft rotation speed. The CT image of the outer clearances shown in (i), (iii), and (v) of Fig. 10, illustrate that the oil film area hardly changed with respect to the change in the shaft rotation speed. On the other hand, in the CT image of the inner clearances (ii), (iv), and (vi) in Fig. 10, the oil film area is gradually decreased as the shaft rotation speed increases. From these results, the cause of suppression of the increase in the bush rotation speed is described as follows: The bush drive torque increases as the shaft rotation speed increases, but the increase is offset by oil film contraction in the inner clearance. On the other hand, as the oil film area of the outer clearance does not change, the bush braking torque has not changed, and the increase in the bush rotation speed is suppressed.

The growth of the gas phase region near the end of the inner clearance observed in the CT images in Fig. 10 is similar to the axial oil film rupture phenomenon confirmed by the theoretical predictions by Hatakenaka [12] et al. and oil film observation by Tanaka [13]. However, according to Tanaka, when this phenomenon is observed, the shaft velocity of the plain bearing is approximately $31 \mathrm{~m} / \mathrm{s}$, which is approximately three times the rotation speed at the time of oil film observation in this study. Therefore, the contraction in the oil film area observed in this study is different from the previously reported axial oil film rupture phenomenon. In this study, the cause of oil film contraction is that as the rotation speed of the shaft increases, the centrifugal force acting on the oil film in the inner clearance increases. This centrifugal force prevents the oil from flowing from the outer clearance into the inner clearance, reducing the amount of oil flowing in and the oil film in the inner clearance.

3.3 Relationship between oil film area and floating bush speed

In the floating bush bearing, oil did not flow into the inner clearance from the oil port at any shaft rotation speed. Therefore, the oil film area and bush rotation speed are different from the case in which the oil flows into the inner clearance from the oil port. Thus, after confirming that the oil flowed into the inner clearance from the oil port during the oil film observation, the rotation speed of the bush was measured, and the result was compared with the above result.

As a method of flowing oil from the oil port to the inner clearance, it is conceivable to increase the oil supply pressure. However, this method was not suitable because the oil scatter in the X-ray CT machine. Thus, an oil groove with a depth of $500 \mu \mathrm{m}$ was machined in the circumferential direction at the center of the width direction of the floating bush. As an effect of this machining, the supplied oil does not spread in the bearing width direction and instead flows into the oil groove, after which it can flow into the inner clearance.

Figure 11 shows the 3D CT image of the grooved floating bush bearing. This CT image was taken at a shaft rotation speed of 10,000 rpm. The voxel size of this CT image was $30 \mu \mathrm{m}$, size of the focal spot on the X-ray port was $27 \mu \mathrm{m}$, and a $2.5 \mathrm{~mm}$ thick copper filter was attached to the $X$-ray port. This image shows that oil flows from the oil port into the inner clearance by machining the oil groove, and that the oil film area increases more in the inner clearance compared to the bush before a groove was machined.

Figure 12 shows the measured bush rotation speed of the grooved floating bush bearing. As the shaft rotation speed increases, the bush rotation speed continues to increase, and a region in which the bush rotation speed becomes constant cannot be confirmed. Furthermore, the slope of the increase in bush rotation speed is also larger than that of the bush without grooves.

The results above indicate that, the bush rotation speed largely depends on the oil film area of the inner clearance.

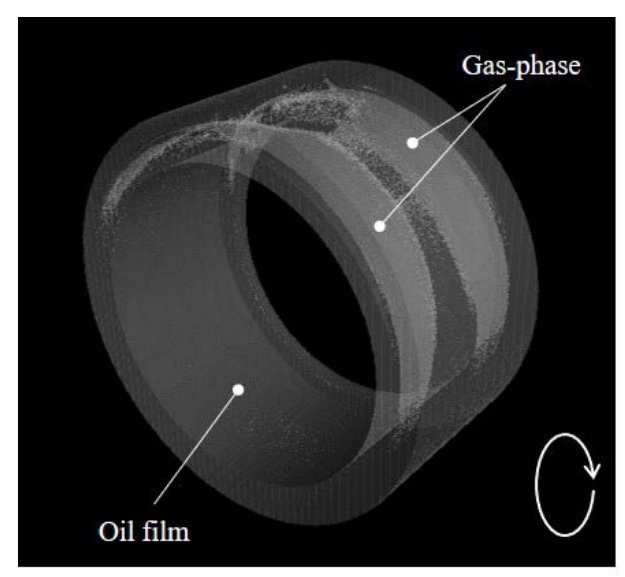

(i) Outer clearance

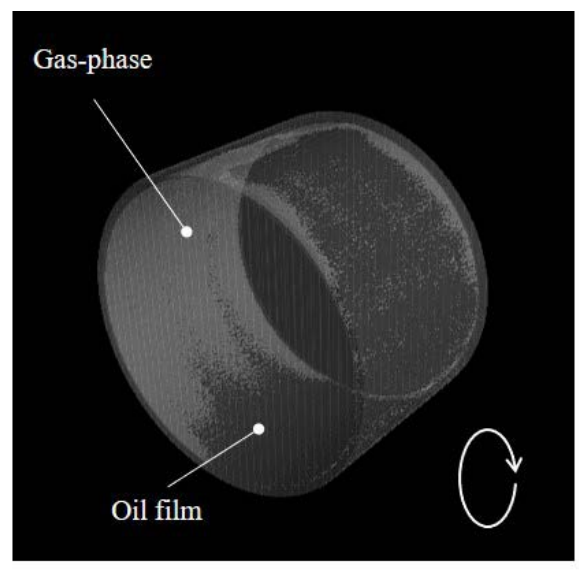

(ii) Inner clearance

Fig. 11 3D CT image of a grooved floating bush bearing whose shaft rotates at 10,000 rpm 


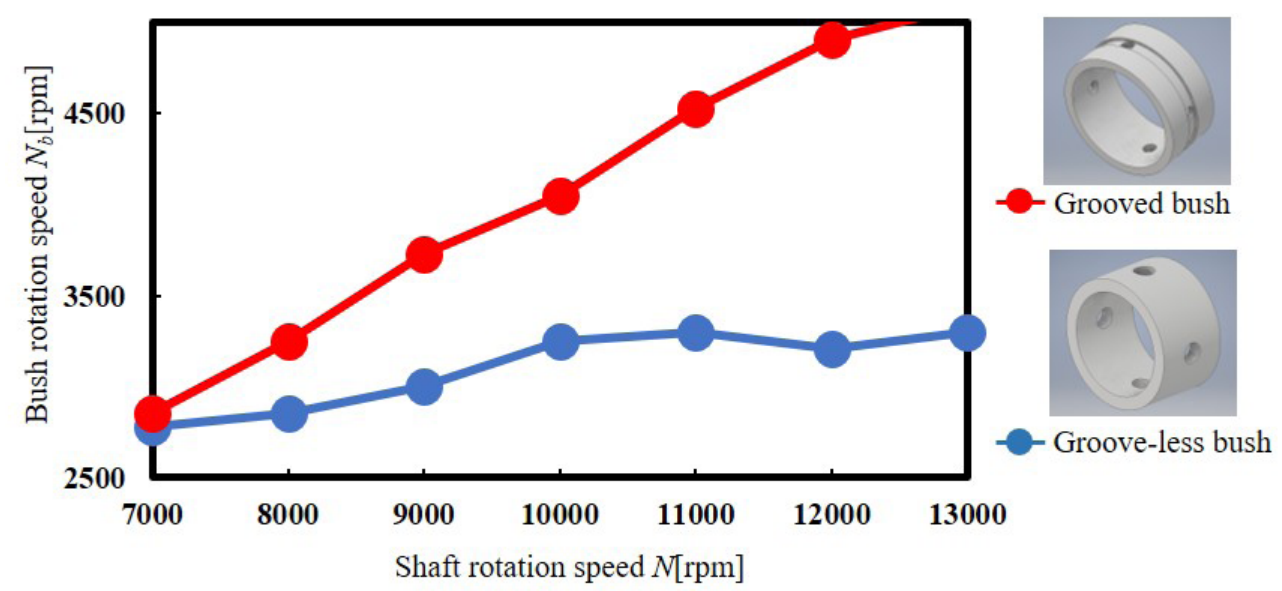

Fig. 12 Measured bush rotation speed of a grooved floating bush bearing

Additionally, when the oil is sufficiently supplied to the inner clearance, the increase in the bush rotation speed is not suppressed.

\section{Conclusion}

In this study, the bush rotation speed and oil film area were compared by an observation of the oil film using X-ray CT. This clarified why the increase in the bush rotation speed was suppressed, despite the increase in shaft rotation speed. The results are as follows:

(1) The double oil film of a floating bush bearing can be observed by X-ray CT.

(2) The oil film area in the inner clearance decreases when the increase in the bush rotation speed is suppressed. On the other hand, no significant change is observed in the oil film area of the outer clearance. This phenomenon can rationally explain why the increase in the bush rotation speed was suppressed despite an increase in the shaft rotation speed.

(3) The bush rotation speed increases linearly with the increase in the shaft rotation speed when the oil film area of the inner clearance increases. Therefore, it is considered that the suppression of the increase in the bush rotation speed does not occur if the oil is sufficiently flowing into the inner clearance.

\section{Acknowledgment}

This work is partially supported by Tokai University Imaging Center for Advanced Research. In particular, Ms. Awano of Micro-Nano Center received immense support, which we gratefully acknowledge.

\section{References}

[1] Tanaka, M. and Hori, Y., "Stability Characteristics of Floating Bush Bearings," Journal of Lubrication Technology, 94, 3, 1972, 248-256.

[2] Tanaka, M. and Hatakenaka, K., "A Stability Analysis of HighSpeed Floating Bush Journal Bearings," Proc. Dynamics and Design Conference, A, 96, 8, 59, 347-350 (in Japanese).

[3] Saito, S., Someya, T. and Nakagawa, E., "Theoretical Investigation into the Stability of a Rotor Supported by Floating Bush Journal Bearings," Transactions of JSME, 40, 338, 1974, 2824-2831 (in Japanese)

[4] Yoshioka, K., "Study on the Floating-Bush Journal Bearing (1),"
Journal of Japanese Society of Lubrication Engineers, 16, 7, 1971, 509 (in Japanese).

[5] Yoshioka, K., "A Study of the Bush Speed Raito of a Floating-Bush Bearing," Journal of Japanese Society of Lubrication Engineers, 19, 3, 1974, 217-221 (in Japanese).

[6] Orcutt, F. K. and Ng, C. W., "Steady-State and Dynamic Properties of the Floating-Ring Journal Bearing," Journal of Lubrication Technology, 90, 1, 1968, 243-253.

[7] Hatakenaka, K., Tanaka, M. and Suzuki, K., "A Modified Reynolds Equation with Centrifugal Force Being Considered and Its Application to Floating Bush Bearing," Transactions of the JSME, Part C, 65, 636, 1999, 3395-3400 (in Japanese).

[8] Trippett, R. J. and Li, D. F., "High-Speed Floating-Ring Bearing Test and Analysis," ASLE Transactions, 27, 1, 1984, 73-81.

[9] Trippett, R. J., "Measured and Predicted Friction in Floating-Ring Bearings," SAE Transactions, 1986, 470-476.

[10] Tatara, A., "An Experimental Study of the Stabilizing Effect of Floating-Bush Journal Bearings," Bulletin of JSME, 13, 61, 1970, 858863.

[11] Tsuruta, Y. and Tsuda, K., "Investigation into an Effect of Floating Bush Bearing, in Suppressing Oil-Whip at Higher Shaft Speed," Journal of Japanese Society of Lubrication Engineers, 30, 11, 1985, 838-845 (in Japanese).

[12] Hatakenaka, K., Tanaka, M. and Suzuki, K., "A Theoretical Analysis of Floating Bush Journal Bearing with Axial Oil Film Rupture Being Considered," Journal of Tribology, 124, 3, 2002, 494-505.

[13] Tanaka, M., "Axial Oil Film Rupture in High Speed Plain Bearings," Proc. Tribology Conference Tokyo 2000, Tokyo, 2000, 227-278 (in Japanese).

[14] Yan, W., Dong, R. X., Song, L. X. and Wei, G. C., “Numerical Investigation of Air-Oil-Thermal Coupling Mechanism in Floating Ring Bearings," Journal of Tribology, 140, 3, 2018, 031701.

[15] Tsuda, K., Takahashi, T. and Tsurumaki, N., “Observation of Oil Film Disappearance between Shaft and Fast Rotating Bush Lubricated from Outside," Journal of Japanese Society of Lubrication Engineers, 30, 1, 1985, 69-72 (in Japanese).

[16] Sakai, F., Ochiai, M. and Hashimoto, H., "Stability Characteristics and CFD Analysis of Two-Phase Flow of Oil Film Journal Bearing Having Two Oil Filler Holes," Transactions of JSME, 83, 848, 2017, 16-00457 (in Japanese).

[17] Cole, J. A. and Hughes, C. J., “Oil Film Extent in Complete Journal Bearings," Industrial Lubrication and Tribology, 7, 6, 1955, 16-22.

[18] Otsu, T. and Nishida, K., "Growth of Cavitation in Journal Bearing and Effect of Cavitation on Behavior of Bearing," Japanese Society of Tribologists, 61, 2, 2016, 127-136 (in Japanese). 